\title{
Hubert John und Philipp Maletzki Vom Frust zur Lust in der Urologie. Zur Behandlung von Harninkontinenz und erektiler Dysfunktion
}

Haben Sie auch schon einmal beim Heben einer schweren Getränkekiste einige Tropfen Urin verloren? Oder kennen Sie vielleicht jede öffentliche Toilette in der Innenstadt und verlieren Urin bei starkem Harndrang? Dann könnte bei Ihnen eine Belastungs- oder Dranginkontinenz vorliegen! Und wenn es beim Geschlechtsakt hakt, weil der Penis nicht genügend hart wird, dann ist der Frust komplett!

Harninkontinenz und erektile Dysfunktion sind nach wie vor Tabuthemen, die nicht gerne verbalisiert werden. Sie gehen mit Schamgefühl, Unwohlsein und Unsicherheit einher und werden daher nicht gern in der Öffentlichkeit, im Privaten oder auch beim Arztbesuch angesprochen. Harninkontinenz ist eine häufige Erkrankung, die Frauen und Männer mit zunehmendem Alter betreffen kann. Bei Frauen kann sie schon ab dem 30. Lebensjahr auftreten, bei Männern tritt sie oft erst ab dem 50. Lebensjahr auf. Je nach Literatur leiden zwischen $30 \%$ und $60 \%$ der 80 -Jährigen beider Geschlechter an einer Form der Harninkontinenz. ${ }^{1}$

1 Alayne Markland et al.: Prevalence of urinary incontinence in men: results from the national health and nutrition examination survey, in: Journal of Urology 184 (2010) 1022; Dina Bedretdinova et al.: Prevalence of Female Urinary Incontinence in the General Population According to Different Definitions and Study Designs, in: European Urology 69 (2016) 256; Louise Schreiber Pedersen et al.: Prevalence of urinary incontinence 
182 Hubert John und Philipp Maletzki: Vom Frust zur Lust in der Urologie

Die Prävalenz der erektilen Dysfunktion liegt je nach Studienpopulation bei ca. 20\%, wobei ein altersbedingter Anstieg von ca. $2 \%$ bei den 30 -Jährigen bis zu über $50 \%$ bei den 80 -Jährigen nachgewiesen werden konnte. ${ }^{2} 25 \%$ der Männer, die erstmals einen Arzt wegen erektiler Dysfunktion aufsuchen, sind unter 40 Jahre alt. $^{3}$

\section{Der erste Schritt - Vertrauen schaffen}

Nicht nur Patienten mit Harninkontinenz oder erektiler Dysfunktion können von Frust geplagt sein - auch der behandelnde Arzt. Die Abklärung und Behandlung dieser Erkrankungen ist oft aufwendig, zeitintensiv und kann frustrierend sein, wenn sich der gewünschte Therapieerfolg (noch) nicht einstellt oder nicht einstellen wird.

Grundvoraussetzung einer guten Arzt-Patienten-Beziehung ist eine gemeinsame Vertrauensbasis. So können Schamgefühle überwunden und intime Probleme, die sonst im Verborgenen bleiben würden, verbalisiert werden. Auch gehört es zur Aufgabe des Arztes, die möglicherweise schamhaften Umschreibungen des Patienten richtig zu deuten, die Beschwerden zu erfassen und die Symptomatik offen anzusprechen. Auf einer guten Vertrauensbasis gestaltet sich der Weg zur Therapie einfacher.

among women and analysis of potential risk factors in Germany and Denmark, in: Acta Obstetricia et Gynecologica Scandinavica 96/ 8 (2017) 939-948.

2 Moritz Braun et al.: Epidemiology of erectile dysfunction: results of the 'Cologne Male Survey', in: International Journal of Impotence Research 12 (2000) 305.

3 Paul Capogrosso et al.: One patient out of four with newly diagnosed erectile dysfunction is a young man - worrisome picture from the everyday clinical practice, in: The Journal of Sexual Medicine 10 (2013) 1833. 
183 Hubert John und Philipp Maletzki: Vom Frust zur Lust in der Urologie

\section{Harninkontinenz}

Harninkontinenz ist nicht gleich Harninkontinenz. - Grundsätzlich handelt es sich bei der Harninkontinenz um einen ungewollten Urinverlust, der ein soziales oder ein hygienisches Problem darstellt. Die ärztliche Abklärung hat zunächst das Ziel, die Art der Inkontinenz zu diagnostizieren. So kann eine Belastungsinkontinenz vorliegen, wenn es zu Urinverlust beim Heben, Husten, Niessen oder Gehen kommt. Dies ist mit über 50\% die häufigste Inkontinenzform bei Frauen. Sie tritt jedoch auch bei Männern auf, und zwar vor allem nach operativer Prostataentfernung bei einer Prostatakarzinomerkrankung - die Rate liegt hier bei $4 \%$ bis 35\% der Fälle. ${ }^{4}$ Die Ursache einer Belastungsinkontinenz ist oft eine Beckenboden- oder Bindegewebsschwäche, die altersbedingt oder durch vorausgehende Operationen oder Geburten ausgelöst worden sein kann. Eine Schliessmuskelschwäche wird zusätzlich durch die Menopause begünstigt. Weiterhin kann ein erhöhter Bauchdruck bei Asthma, Raucherhusten oder Übergewicht zu einer Belastungsinkontinenz führen.

Kommt es zu plötzlich einsetzendem Harndrang mit unkontrolliertem Urinverlust, so liegt eine Dranginkontinenz vor. Dies ist bei Männern die häufigste Inkontinenzform (bis zu 80\% der Fälle). Einer Dranginkontinenz kann eine Harnwegsinfektion zugrunde liegen, oder sie kann Folge einer gutartigen Prostatavergrösserung sein. Auch Tumore des Harntraktes können Drangbeschwerden hervorrufen. Weiter können Erkrankungen des Nervensystems (wie Demenz, Morbus Alzheimer, Parkinson oder Rückenmarksverletzungen) zu Drangbeschwerden führen.

Abklärung in Schritten. - Am Anfang einer Abklärung steht ein ausführliches Arzt-Patienten-Gespräch, in dem die Beschwerden erfasst und eingeordnet werden. Ergänzend werden Fragebögen und Miktionstagebücher eingesetzt, durch die die Symptome objektiviert werden sollen. Zur Basisdiagnostik gehört sodann die Untersuchung

$4 \quad$ Vincenzo Ficarra et al.: Systematic Review and Meta-analysis of Studies Reporting Urinary Continence Recovery After Robot-assisted Radical Prostatectomy, in: European Urology 62 (2012) 405. 
184 Hubert John und Philipp Maletzki: Vom Frust zur Lust in der Urologie

des Genitaltraktes und eine Urinuntersuchung zum Ausschluss einer Harnweginfektion.

Die apparative urologische Untersuchung - ein weiterer Schritt umfasst eine Harnstrahlmessung, aus der pathologische Harnflussmuster abgeleitet werden können, sowie eine Ultraschalluntersuchung. Letztere dient der Diagnostik von Rest-Urin, Prostatagrösse und Blasentumoren. Mittels einer Blasenspiegelung (Zystoskopie) können morphologische Aspekte der Harnröhre (Harnröhrenverengung), Prostata (Prostatavergrösserung) und Harnblase (Blasentumore, Blasensteine, Blasendivertikel) diagnostiziert werden (Abb. 1).

\section{Erektile Dysfunktion}

Wann liegt eine erektile Dysfunktion vor? - Unter erektiler Dysfunktion versteht man die Unfähigkeit, eine Erektion zu erhalten oder aufrecht zu erhalten, um einen zufriedenstellenden Geschlechtsverkehr ausüben zu können. Eine Erektionsstörung kann daher neben der körperlichen Auswirkung auch einen starken Einfluss auf die psychische Gesundheit des Mannes und die Partnerschaft haben und damit die Lebensqualität wesentlich beeinträchtigen.

Für die Erektion ist ein komplexes Zusammenspiel aus neurologischen, gefäss- und gewebsspezifischen Faktoren notwendig. Ursachen einer erektilen Dysfunktion können Gefässveränderungen, etwa im Rahmen von Arteriosklerose, Diabetes mellitus, Bluthochdruck oder als Folge von Nikotinkonsum sein. Auch eine Nervenschädigung im Rahmen eines Schlaganfalls, einer Rückenmarksverletzung oder nach einer Operation kann eine Erektionsstörung begünstigen. Bei psychischer Belastung kann es zu einer psychosomatischen Erektionsstörung kommen. Darüber hinaus können hormonelle Störungen (wie ein ausgeprägter Testosteronmangel) zu erektiler Dysfunktion führen.

Schritte der Abklärung. - Zunächst wird im Rahmen eines ausführlichen Gesprächs eine Sexualanamnese durchgeführt, in der sowohl der Beginn der Erektionsstörung, vorausgehende Konsultationen 
185 Hubert John und Philipp Maletzki: Vom Frust zur Lust in der Urologie

oder Therapieversuche als auch das subjektive Lustempfinden (Libido) und die objektive Orgasmus-Fähigkeit erfasst werden. Begleitend ist eine ausführliche Anamnese der Partnerschaft im Hinblick auf mögliche Konflikte, des allgemeinen physischen und psychischen Gesundheitsstatus, des Medikamentengebrauchs und des Einsatzes von Noxen (Nikotin, Alkohol und Drogen) wichtig. Auch Faktoren, die auf eine Hormonstörung hindeuten, wie beispielsweise vermehrte Müdigkeit, Energielosigkeit und Konzentrationsstörungen, müssen erfragt werden. Zur Objektivierung der Beschwerden wird ein Fragebogen eingesetzt.

Neben der Untersuchung des Genitalstatus (Hodengrösse, Hodenveränderungen, Penisdeformitäten und Prostataauffälligkeiten) wird auch hormonellen Aspekten und den sekundären Geschlechtsmerkmalen (Behaarungstyp, Körperbau) Beachtung geschenkt. Die weitere Abklärung umfasst eine Blutuntersuchung, in der das Testosteron sowie die Blutfett- und Blutzuckerwerte bestimmt werden.

\section{Therapie}

Der erste Therapiebaustein sowohl bei Harninkontinenz als auch bei erektiler Dysfunktion ist eine ausführliche Aufklärung und Information über die Erkrankung. Hierbei sollten die Erwartungen und Bedürfnisse der Patienten erörtert werden. Bei einer erektilen Dysfunktion muss der Patient darüber aufgeklärt werden, dass diese lediglich ein Symptom einer anderen Krankheit ist. Auch wenn die zugrundeliegende Krankheit in vielen Fällen erfolgreich behandelt werden kann, ist nicht ausgeschlossen, dass die erektile Dysfunktion als Symptom bestehen bleibt.

Zur Basistherapie beider Störungen wird eine gesunde Lebensweise empfohlen, die regelmässige körperliche Aktivität, Gewichtsreduktion, gesunde Ernährung sowie Alkohol- und Nikotinverzicht umfasst. 
186 Hubert John und Philipp Maletzki: Vom Frust zur Lust in der Urologie

\section{Harninkontinenz}

Konservative Massnahmen. - Bei einer Belastungsinkontinenz wird die Durchführung eines physiotherapeutisch begleiteten Beckenbodentrainings empfohlen, das durch Hilfsmittel, die manuell Nichtmessbares apparativ messbar machen sollen (Biofeedback), ergänzt werden können. Auch die Dranginkontinenz kann je nach zugrundeliegender Blasenfunktionsstörung mittels Physiotherapie sehr gut behandelt werden. Weiter kann eine Pessar-Anpassung erfolgen. Durch mechanischen Druck auf die Harnröhre und Optimierung der Position des Beckenbodens kann die Harninkontinenz reduziert werden (Abb. 2).

Medikamentöse Therapie. - Vor allem bei Dranginkontinenz spielt die medikamentöse Therapie eine wichtige Rolle. Hierbei kommen Medikamente (Antimuskarinika und/oder Sympathomimetika) zum Einsatz, die die Empfindlichkeit der Harnblase dämpfen und so die Häufigkeit und den Drang vor dem und beim Wasserlösen senken. Als Nebenwirkungen können Mundtrockenheit oder Verstopfung auftreten, über die es zuvor aufzuklären gilt. Weiter kommt bei der Dranginkontinenz die Injektion von Onabotulinumtoxin A (Botox $\left.{ }^{\circledR}\right)$ in den Harnblasenmuskel zum Einsatz, wodurch die Blasenüberaktivität gesenkt wird und die Drangbeschwerden eine gewisse Zeit nachlassen.

Elektrische interventionelle Therapie. - Liegt eine Dranginkontinenz vor, die nicht auf konservative oder medikamentöse Therapien anspricht, kann alternativ eine perkutane oder transkutane Nervenstimulation erfolgen. Durch Stimulation des Nervus tibialis posterior, der im Fussknöchelbereich verläuft, können indirekt Nervenzentren, die für die Blasenfunktion zuständig sind, moduliert werden.

Bei ungenügendem Therapieerfolg kann auch ein Blasenschrittmacher unter die Haut implantiert werden, die sogenannte sakrale Neuromodulation. Über mehrpolige Elektroden werden die Nervenwurzeln moduliert, aus denen auch die Blasennerven kommen. Durch dieses ambulante Verfahren können Drangbeschwerden in 
187 Hubert John und Philipp Maletzki: Vom Frust zur Lust in der Urologie

bis zu 80\% der Fälle verbessert werden. Der genaue Wirkmechanismus der Modulation der Nerven ist jedoch nicht bekannt.

Operative Verfahren. - Sowohl bei Belastungs- als auch bei Dranginkontinenz kommen operative Verfahren zum Einsatz. So kann bei Belastungsinkontinenz bei Frauen wie Männern eine Band-Einlage im Bereich der Harnröhre erfolgen, um den Auslasswiderstand des Wasserlösens zu erhöhen. Die Einlage eines solchen TVT (transvaginales Tape) oder TOT (transobturatorisches Tape) bei Frauen kann die Belastungsinkontinenz zu 80\% verbessern. ${ }^{5}$ Das männliche Pendant, die bulbourethrale Urethrasuspension, hat eine Erfolgsrate von ca. 65\% mit ebenso deutlicher Verbesserung der Lebensqualität. ${ }^{6}$ Das Standardverfahren stellt die Implantation eines künstlichen Harnröhrenschliessmuskels (AMS 800, ATOMS oder Zephyr) dar - hier liegen die Kontinenzraten bei bis zu 80\% (Abb. 3).

Zur Behandlung von irritativen Drangbeschwerden aufgrund einer vorliegenden gutartigen Prostatavergrösserung wird als Standardverfahren die transurethrale Resektion der Prostata (TUR-P) angewandt. Hierbei wird mittels elektrischer Schlinge, die unter Kamerasicht über die Harnröhre eingeführt werden kann, Prostatagewebe schichtweise abgetragen.

\section{Erektile Dysfunktion}

Konservative Massnahmen. - Die Therapieentscheidung sollte darauf basieren, ob Risikofaktoren oder begünstigende Grunderkrankungen zur Entwicklung einer erektilen Dysfunktion vorliegen, die es primär zu behandeln gilt. Erst sekundär oder parallel hierzu sollten ergänzende Therapiemassnahmen zum Einsatz kommen. Im Falle von prädisponierenden Risikofaktoren (Blutincontinence, in: European Urology 62 / 6 (2012) 1118-1129.

6 Hubert John et al.: Bulbourethrale kombinierte Urethrasuspension: Alternative zur AMS-Sphinkterprothese, in: Journal für Urologie und Urogynäkologie 12 / 4 (2005) 31-34. 
188 Hubert John und Philipp Maletzki: Vom Frust zur Lust in der Urologie

hochdruck, Diabetes mellitus, Übergewicht) sollten diese primär angegangen werden.

Im Falle einer psychosomatischen Komponente oder eines Konflikts innerhalb der Partnerschaft kann es zudem sinnvoll sein, einen Sexual- oder Paartherapeuten zur Behandlung hinzuziehen.

Medikamentöse Therapie. - Mit Viagra ${ }^{\circledR}$, das mit dem Wirkstoff Sildenafil Ende der 1990er Jahre die Marktzulassung erhalten hat, wurde die Therapie der erektilen Dysfunktion revolutioniert. Nachdem die Erforschung von Sildenafil initial der Behandlung von Lungenbluthochdruck galt, zeigte sich als angenehmer Nebeneffekt bei Probanden, dass sich die Erektionsfunktion deutlich verbesserte und viele zuvor impotente Patienten wieder eine Erektion erleben durften. Mittels Einnahme einer Tablette war es nun möglich geworden, eine Erektionsstörung wirkungsvoll zu behandeln. Im Laufe der Jahre wurde weitere Präparate (Cialis ${ }^{\circledR}$, Levitra ${ }^{\circledR}$, Spedra ${ }^{\circledR}$ ) entwickelt, die alle den gleichen Wirkmechanismus mit jedoch unterschiedlicher Wirkdauer aufweisen. Der gemeinsame Angriffspunkt ist ein Enzym namens Phosphodiesterase (PDE)-5, das durch diese Medikamentengruppe gehemmt wird - daher im Arztjargon auch «PDE-5-Hemmer» genannt. PDE-5-Hemmer bewirken im Schwellkörpergewebe die Relaxation der glatten Muskulatur mit resultierendem verbessertem arteriellem Bluteinfluss und folgender Erektionsverbesserung.

Damit aus Frust Lust werden kann, muss eine korrekte Anwendung erfolgen. Das Originalpräparat sollte ca. 30-60 Minuten vor dem geplanten Geschlechtsverkehr eingenommen werden. Ein Wechsel des Präparats oder die Steigerung der Dosis sollte frühestens nach sechs Versuchen ins Auge gefasst werden. Damit das Präparat eine erfolgreiche Wirkung zeitigt, bedarf es externer (taktiler, visueller) Stimulation und / oder interner (psychischer) Disposition. Die Erfolgsrate aller Präparate liegt durchschnittlich bei ca. 80\%.7 Als Nebenwirkungen können Kopfschmerzen oder plötzliche Gesichtsröte auftreten. Eine Anwendungsbeschränkung gilt bei kürzlich

7 Irwin Goldstein et al.: Oral sildenafil in the treatment of erectile dysfunction. 1998, in: The Journal of Urology 167 (2002) 1197. 
stattgehabtem Herzinfarkt, Schlaganfall, deutlich erniedrigtem Blutdruck und schwerer Herzschwäche.

Eine weitere Option stellt die Schwellkörperautoinjektionstherapie (SKAT) dar. Hierbei appliziert sich der Patient selbstständig mittels Spritze ein gefässerweiterndes Medikament direkt in den Schwellkörper. Durch dieses Verfahren wird eine Erektionsquote von $90 \%$ erreicht. $^{8}$

Technische Hilfsmittel. - Bringt die medikamentöse Therapie nicht den gewünschten Erfolg oder liegen Erkrankungen vor, die einen Medikamenteneinsatz verbieten, können alternative Hilfsmittel beigezogen werden. Eine Vakuumpumpe als Erektionshilfe war lange Zeit das Standardverfahren. Auch heute noch kommt dieses Gerät, das technisch deutlich weiterentwickelt wurde, zum Einsatz (Abb. 4). Eine geschlechtsverkehrstaugliche Erektion kann in bis zu 90\% der Fälle erreicht werden.

Operative Verfahren. - Ein aufwendigeres Verfahren zur dauerhaften Behandlung einer erektilen Dysfunktion stellt die operative Implantation einer Penisprothese dar (Abb. 5). Diese kann entweder semirigide sein, wobei der Penis mit der «halbsteifen» Prothese vor dem Geschlechtsverkehr nach oben «geklappt» wird, oder aus einem rigiden System bestehen, das heisst aus einem mit Flüssigkeit gefüllten Ballonreservoir, das im Unterbauch des Patienten implantiert wird, einer Pumpe, die im Hodensackbereich platziert wird und einem Schwellkörperzylinderpaar, das im Penis zu liegen kommt. Durch manuelles Betätigen der Pumpe resultiert eine Flüssigkeitsumverteilung aus dem Ballonreservoir in das Zylinderpaar, und es kommt zur Erektion. Die Zufriedenheitsrate liegt bei $92 \%$ bis $100 \% .{ }^{9}$

Hartmut Porst et al.: SOP conservative (medical and mechanical) treatment of erectile dysfunction, in: The Journal of Sexual Medicine 10 (2013) 130. complications with 253 implants in Italy and Germany, in: The Journal of Sexual Medicine 5 (2008) 1503. 
190 Hubert John und Philipp Maletzki: Vom Frust zur Lust in der Urologie

Zusammenfassend ist zu sagen, dass weder über die Harninkontinenz noch über die erektile Dysfunktion ein Mantel des Schweigens ausgebreitet werden sollte. Diese Erkrankungen können frustrierend sein und stellen je nach Patient eine starke psychosoziale Belastung dar, wenn man keine ärztliche Hilfe sucht. Doch nicht nur die Patientin oder der Patient, auch Hausärztinnen und Fachärzte müssen sensibilisiert werden, die Anzeichen einer Harninkontinenz oder Erektionsstörung zu erkennen und offen anzusprechen. Denn erfreulicherweise gibt es viele Behandlungsansätze - konservativ, medikamentös oder operativ - damit aus Frust wieder Lust werden kann. 


\section{Abbildungen}

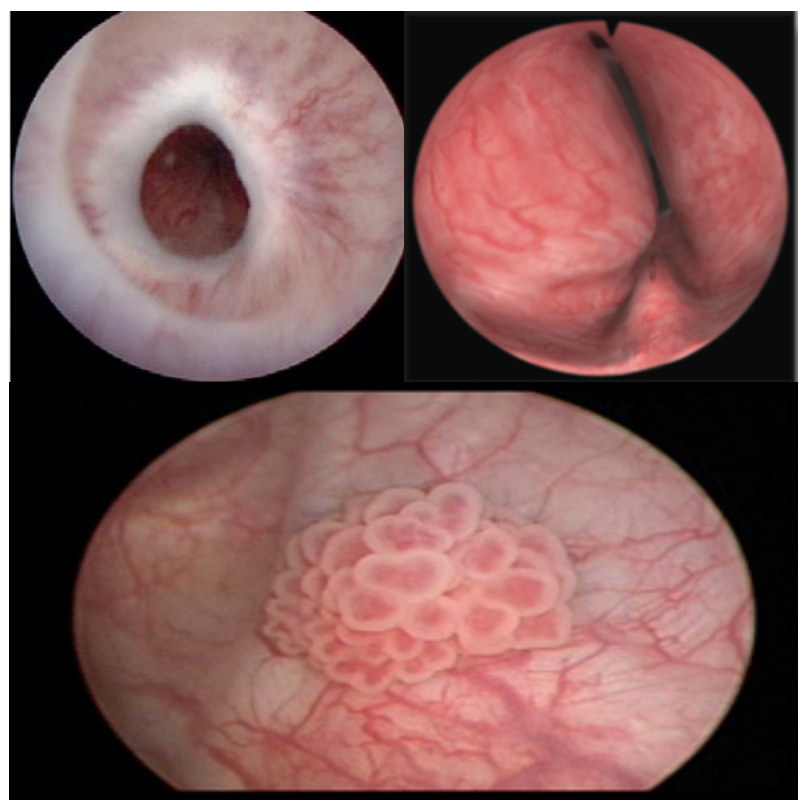

Abb. 1: Nachweis einer Harnröhrenverengung (oben links), ${ }_{1}^{1}$ Prostatavergrösserung (oben rechts) ${ }^{2}$ und eines Blasentumors (unten) ${ }^{3}$

https://www.urologielehrbuch.de/harnroehrenstriktur 02.html http://www.okbmedical.com/turpsim.html

${ }^{3}$ http: / / www.praxis-dr-kuehne.de/leistungen/trus/

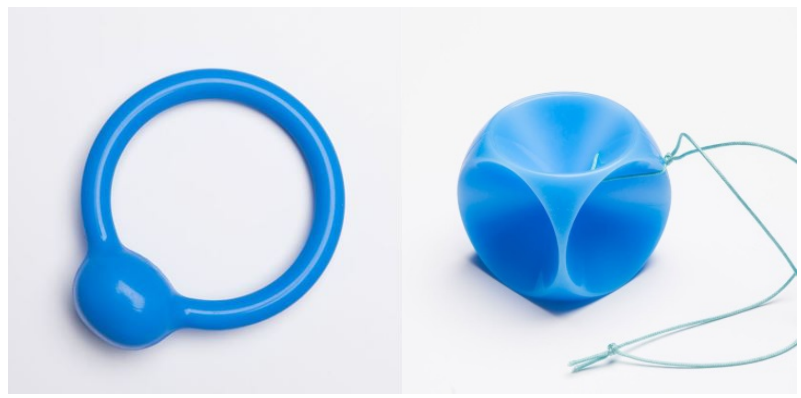

Abb. 2 Ring- und Würfelpessar zur Repositionierung der Harnröhre und des Beckenbodens

Dr. Arabin GmbH \& Co. KG, Witten, Deutschland 
192 Hubert John und Philipp Maletzki: Vom Frust zur Lust in der Urologie
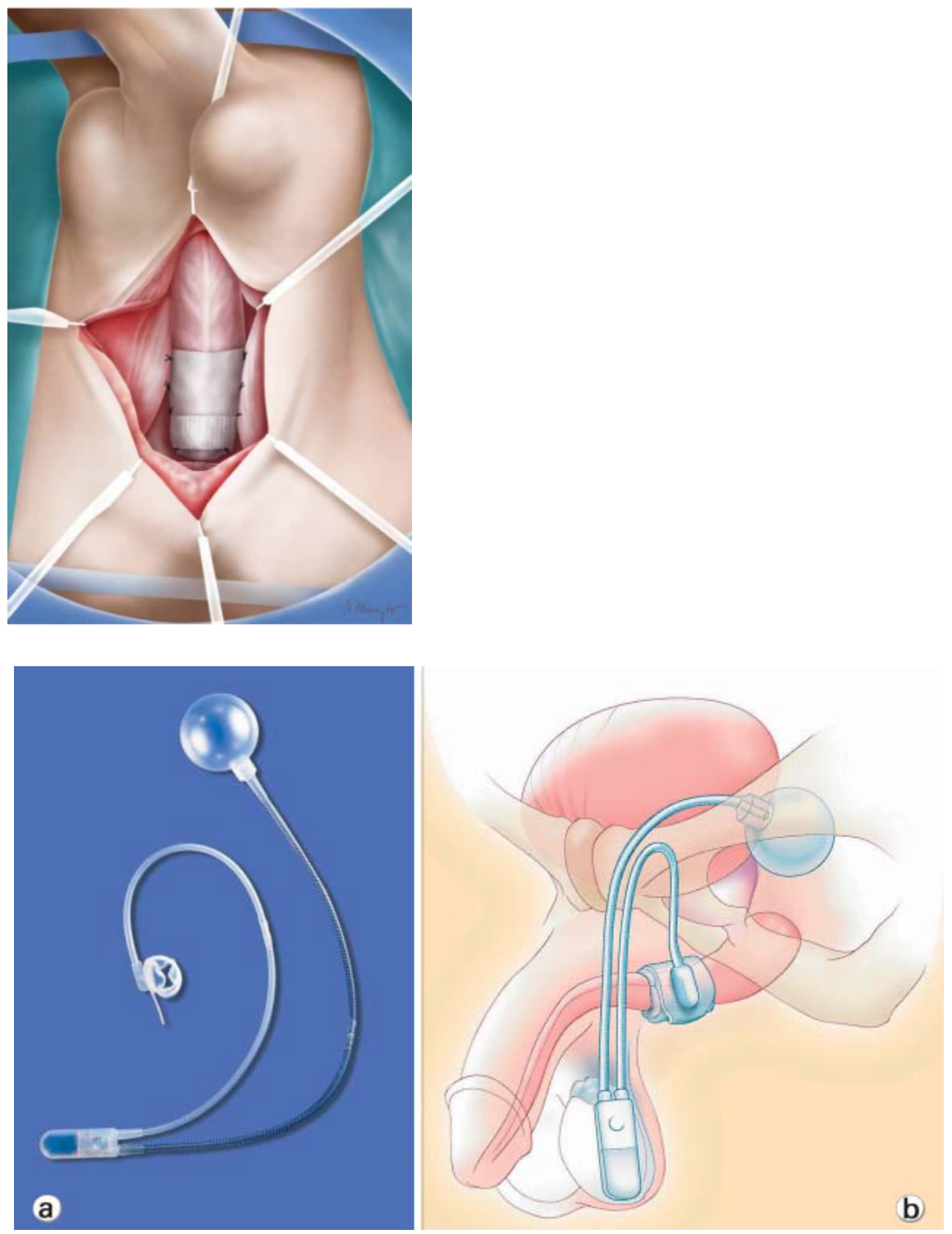

Abb. 3: Band-Einlage beim Mann

Oben: Bulbourethrale Urethra-Suspension

Unten: Implantation eines künstlichen Harnröhrenschliessmuskels

Hubert John et al.: Bulbourethrale kombinierte Urethrasuspension: Alternative zur AMSSphinkterprothese, in: Journal für Urologie und Urogynäkologie 12 / 4 (2005) 31-34. 
193 Hubert John und Philipp Maletzki: Vom Frust zur Lust in der Urologie

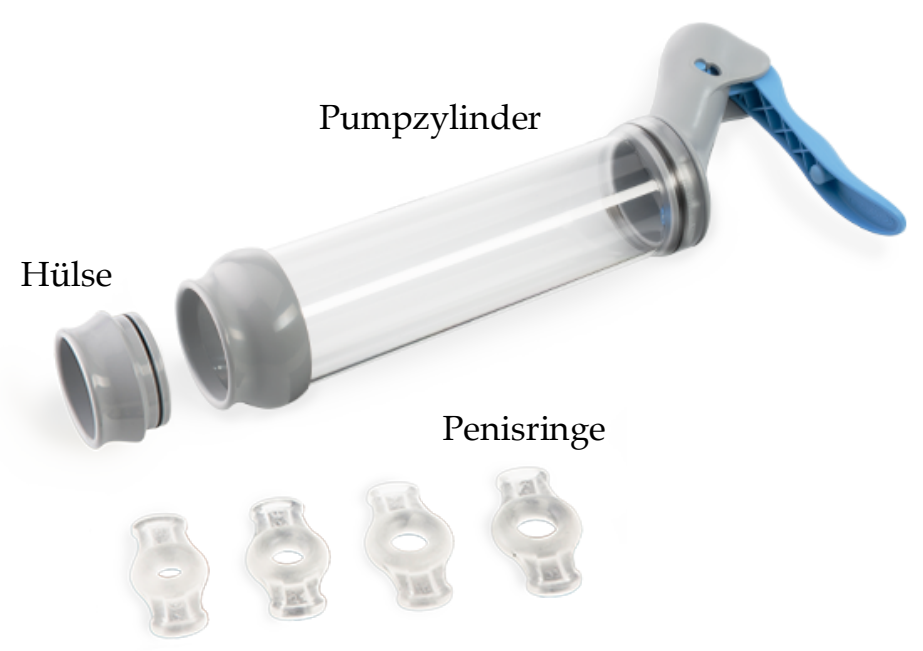

Abb. 4: Vakuum-Erektionshilfe

MANUAL Erection System ${ }^{\circledR}$, Kessel medintim GmbH, Walldorf, Deutschland 
194 Hubert John und Philipp Maletzki: Vom Frust zur Lust in der Urologie

\section{Ballonreservoir}

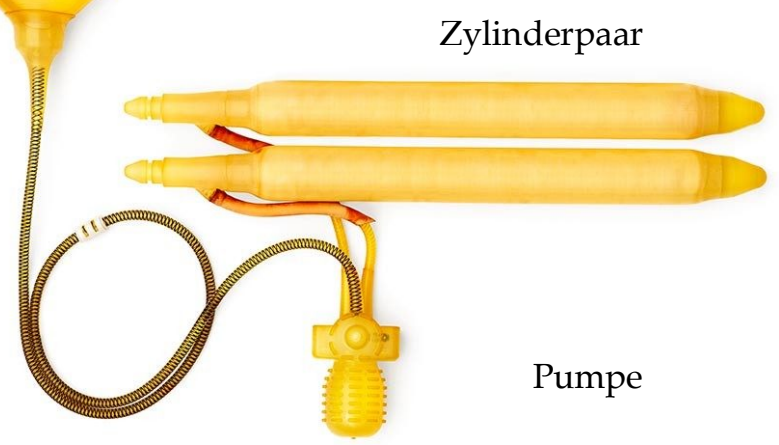

Abb. 5: Penisprothese zur Erektionsbehandlung

American Medical Systems (AMS) $700{ }^{\circledR}$, Boston Scientific, Marlborough, MA, USA

conexus 1 (2018) 181-194

(C) 2018 Hubert John und Philipp Maletzki. Dieser Beitrag darf im Rahmen der Lizenz CC BY-NC-ND 4.0 - Creative Commons: Namensnennung/ nicht kommerziell/keine Bearbeitungen - weiterverbreitet werden.

\section{(c) $(1)(8)$}

https:/ / doi.org/10.24445/ conexus.2018.01.012

Prof. Dr. Hubert John, Kantonsspital Winterthur, Brauerstrasse 15, 8401 Winterthur

hubert.john@ksw.ch

Dr. Philipp Maletzki, Kantonsspital Winterthur, Brauerstrasse 15, 8401 Winterthur philipp.maletzki@ksw.ch 